\title{
A Study of Double Euler Summability Method of Fourier Series and its Conjugate Series
}

\author{
Kalpana Saxena ${ }^{1}$, Manju Prabhakar ${ }^{2}$ \\ ${ }^{1}$ Department of Mathematics, Govt. M.V.M. Bhopal, India \\ ${ }^{2}$ Research Scholar, Bhopal, India \\ Corresponding address: manjuprabhakar2@gmail.com
}

Abstract: In this paper, the product of Euler means is taken up to study the double summability of Fourier series and its allied series. We established two new theorems on $(E, 1)(E, 1)$ product summability of Fourier series and its conjugate Fourier series.

Keywords: $(E, q)$ summability, $(E, 1)(E, 1)$ summability.

\section{DEFINITION AND Notation}

Let $f(x)$ be a $2 \pi$ periodic function and Lebesgue integrable over $(-\pi, \pi)$. The Fourier series of $f(x)$ at any point $x$ is given by

$$
\frac{1}{2} a_{0}+\sum_{n=1}^{\infty}\left(a_{n} \cos n x+b_{n} \sin n x\right) \equiv \sum_{n=1}^{\infty} A_{n}(x)
$$

The conjugate series of Fourier series is given by

$$
\sum_{n=1}^{\infty}\left(b_{n} \cos n x-a_{n} \sin n x\right) \equiv \sum_{n=1}^{\infty} B_{n}(x)
$$

We shall use the following notation,

$$
\begin{aligned}
& \phi(t)=f(x+t)+f(x-t)-2 s \\
& \psi(t)=\frac{1}{2}\{f(x+t)-f(x-t)\} \\
& K_{n}(t)=\frac{1}{2^{n+k+1} \pi} \sum_{k=0}^{n}\left(\begin{array}{l}
n \\
k
\end{array}\right)\left\{\sum_{v=0}^{k}\left(\begin{array}{l}
k \\
v
\end{array}\right) \frac{\sin (v+1 / 2) t}{\sin t / 2}\right\} \\
& \tilde{K}_{n}(t)=\frac{1}{2^{n+k+1} \pi} \sum_{k=0}^{n}\left(\begin{array}{l}
n \\
k
\end{array}\right)\left\{\sum_{v=0}^{k}\left(\begin{array}{l}
k \\
v
\end{array}\right) \frac{\cos (v+1 / 2) t}{\sin t / 2}\right\}
\end{aligned}
$$

and

$$
\tau=\left[\frac{1}{t}\right], \text { where } \tau \text { denotes the greatest integer not greater that } \frac{1}{t}
$$

Let $\sum_{n=0}^{\infty} u_{n}$ be a given infinite series with sequence of its $n^{\text {th }}$ partial $\operatorname{sum}\left\{s_{n}\right\}$. The $(E, 1)$ transform is defined as the $n^{\text {th }}$ partial sum of $(E, 1)$ summability and is given by 


$$
t_{n}=\frac{1}{2^{n}} \sum_{k=0}^{n}\left(\begin{array}{l}
n \\
k
\end{array}\right) s_{k}, \text { then }
$$

the infinite series $\sum_{n=0}^{\infty} u_{n}$ is summable to the definite no. $s$ by $(E, 1)(E, 1)$ summability method if

$$
t_{n}^{(E, 1)(E, 1)}=\frac{1}{2^{n}} \sum_{k=0}^{n}\left(\begin{array}{l}
n \\
k
\end{array}\right)\left\{\frac{1}{2^{k}} \sum_{v=0}^{k}\left(\begin{array}{l}
k \\
v
\end{array}\right) s_{v}\right\} \rightarrow s, \text { as } n \rightarrow \infty
$$

\section{INTRODUCTION}

In the field of summability of Fourier series \& its allied series, the product summability $(E, q)(X)$, $(X)(E, q)$ or $|E, q|$ have been studied by a number of researchers like, Mohanty, R. and Mohapatra, S. (1968), Kwee, B. (1972), ${ }^{2}$ Chandra, P. (1977), ${ }^{1}$ Chandra, P. and Dikshit, G.D. (1981), Sachan, M.P. (1983), Bhagwat, Purnima (1987), Nigam, H.K. and Sharma, Ajay (2006), Lal, S., Singh, H.P., Tiwari, ${ }^{8}$ Sandeep kumar, and Bariwal, Chandrashekhar (2010), ${ }^{3}$ Dhakal, Binod Prasad (2011), Rathore, H.L. and Shrivastava, U.K. (2012), Nigam, H.K. and Sharma, K. (2012,2013), Sinha, Santosh kumar \& Shrivastava, U.K. (2014), Mishra, V.N. and Sonavane, Vaishali (2015) and many more, under various type of criteria \& conditions. After this, so many results established on double factorable summability of double Fourier series, the methods of $(C, 1,1),(H, 1,1) \&\left(N, p_{m}, q_{n}\right)$. But yet, no result found on double Euler summability of Fourier series $\&$ its allied series. Under a general condition, hear we have established two new theorems on $(E, 1)(E, 1)$ product summability of Fourier series and its Conjugate series.

\section{MAIN THEOREM}

Theorem 1: Let $\left\{p_{n}\right\}$ be a positive, monotonic, non-increasing sequence of real constants such that

$$
P_{n}=\sum_{v=0}^{n} p_{v} \rightarrow \infty, \text { as } n \rightarrow \infty
$$

If

$$
\Phi(t)=\int_{0}^{t}|\phi(u)| d u=o\left[\frac{t}{\alpha(1 / t)}\right] \text {, as } t \rightarrow+0
$$

where $\alpha(t)$ is positive, non-increasing function of $t$ and $\alpha(n) \rightarrow \infty$, as $n \rightarrow \infty$. Then the Fourier series (1.1) is summable $(E, 1)(E, 1)$ to $f(x)$ at pt $t=x$.

Theorem 2: Let $\left\{p_{n}\right\}$ be a positive, monotonic, non-increasing sequence of real constants such that

$$
P_{n}=\sum_{v=0}^{n} p_{v} \rightarrow \infty, \text { as } n \rightarrow \infty
$$

If

$$
\Psi(t)=\int_{0}^{t}|\psi(u)| d u=o\left[\frac{t}{\alpha(1 / t)}\right], \text { as } t \rightarrow+0
$$

where $\alpha(t)$ is positive, non-increasing function of $t$, then the Conjugate Fourier series (1.2) is summable to 


$$
\tilde{f}(x)=-\frac{1}{2 \pi} \int_{0}^{2 \pi} \psi(t) \cot \left(\frac{t}{2}\right) d t
$$

at every pt, where this integral exists.

\section{LEMMAS}

Lemma 1: For $0 \leq t \leq 1 / n ; \sin (t / 2) \geq t / 2 ; \sin n t \leq n \sin t ;|\cos n t| \leq 1$

$$
\text { Proof: } \begin{aligned}
\left|K_{n}(t)\right| & \leq \frac{1}{2^{n+k+1} \pi}\left|\sum_{k=0}^{n}\left(\begin{array}{l}
n \\
k
\end{array}\right) \sum_{v=0}^{k}\left(\begin{array}{l}
k \\
v
\end{array}\right) \frac{\sin (v+1 / 2) t}{\sin t / 2}\right| \\
& \leq \frac{1}{2^{n+k+1} \pi}\left|\sum_{k=0}^{n}\left(\begin{array}{l}
n \\
k
\end{array}\right) \sum_{v=0}^{k}\left(\begin{array}{l}
k \\
v
\end{array}\right) \frac{(2 v+1) \sin t / 2}{\sin t / 2}\right| \\
& \leq \frac{1}{2^{n+k+1} \pi}\left|\sum_{k=0}^{n}\left(\begin{array}{l}
n \\
k
\end{array}\right)(2 k+1) \sum_{v=0}^{k}\left(\begin{array}{l}
k \\
v
\end{array}\right)\right| \\
& =\frac{1}{2^{n+1} \pi}\left|\sum_{k=0}^{n}\left(\begin{array}{l}
n \\
k
\end{array}\right)(2 k+1)\right| \\
& =\frac{1}{2^{n+1} \pi}(2 n+1) \sum_{k=0}^{n}\left(\begin{array}{l}
n \\
k
\end{array}\right) \\
& =\frac{(2 n+1)}{2 \pi} \\
& =O(n)
\end{aligned}
$$

Lemma 2: For $1 / n \leq t \leq \pi ; \sin (t / 2) \geq t / 2$;

$$
\text { Proof: } \begin{aligned}
\left|K_{n}(t)\right| & \leq \frac{1}{2^{n+k+1} \pi}\left|\sum_{k=0}^{n}\left(\begin{array}{l}
n \\
k
\end{array}\right) \sum_{v=0}^{k}\left(\begin{array}{l}
k \\
v
\end{array}\right) \frac{\sin (v+1 / 2) t}{\sin t / 2}\right| \\
& =\frac{1}{2^{n+k} \pi t}\left[\sum_{k=0}^{n}\left(\begin{array}{l}
n \\
k
\end{array}\right) \sum_{v=0}^{k}\left(\begin{array}{l}
k \\
v
\end{array}\right)\right] \\
& =\frac{1}{2^{n} \pi t}\left[\sum_{k=0}^{n}\left(\begin{array}{l}
n \\
k
\end{array}\right)\right] \\
& =\frac{1}{\pi t} \\
& =O\left(\frac{1}{t}\right)
\end{aligned}
$$

Lemma 3: For $0 \leq t \leq 1 / n, \sin (t / 2) \geq t / 2 ;|\cos n t| \leq 1$

$$
\text { Proof: }\left|\widetilde{K}_{n}(t)\right| \leq \frac{1}{2^{n+k+1} \pi}\left|\sum_{k=0}^{n}\left(\begin{array}{l}
n \\
k
\end{array}\right) \sum_{v=0}^{k}\left(\begin{array}{l}
k \\
v
\end{array}\right) \frac{\cos (v+1 / 2) t}{\sin t / 2}\right|
$$




$$
\begin{aligned}
& =\frac{1}{2^{n+k+1} \pi}\left[\sum_{k=0}^{n}\left(\begin{array}{l}
n \\
k
\end{array}\right) \sum_{v=0}^{k}\left(\begin{array}{l}
k \\
v
\end{array}\right) \frac{|\cos (v+1 / 2)|}{|\sin t / 2|}\right] \\
& =\frac{1}{2^{n+k} \pi t}\left[\sum_{k=0}^{n}\left(\begin{array}{l}
n \\
k
\end{array}\right) \sum_{v=0}^{k}\left(\begin{array}{l}
k \\
v
\end{array}\right)\right] \\
& =\frac{1}{2^{n} \pi t} \sum_{k=0}^{n}\left(\begin{array}{l}
n \\
k
\end{array}\right) \\
& =\frac{1}{\pi t} \\
& =O\left(\begin{array}{l}
1 \\
t
\end{array}\right)
\end{aligned}
$$

Lemma 4: For $1 / n \leq t \leq \pi, \sin (t / 2) \geq t / 2$

$$
\begin{aligned}
& \text { Proof: }\left|\tilde{K}_{n}(t)\right| \leq \frac{1}{2^{n+k+1} \pi}\left|\sum_{k=0}^{n}\left(\begin{array}{l}
n \\
k
\end{array}\right) \sum_{v=0}^{k}\left(\begin{array}{l}
k \\
v
\end{array}\right) \frac{\cos (v+1 / 2) t}{\sin t / 2}\right| \\
& \leq \frac{1}{2^{n+k} \pi t}\left|\sum_{k=0}^{n}\left(\begin{array}{l}
n \\
k
\end{array}\right) \sum_{v=0}^{k}\left(\begin{array}{l}
k \\
v
\end{array}\right) \cos (v+1 / 2) t\right| \\
& \leq \frac{1}{2^{n+k} \pi t}\left|\sum_{k=0}^{n}\left(\begin{array}{l}
n \\
k
\end{array}\right) \operatorname{Re}\left\{\sum_{v=0}^{k}\left(\begin{array}{l}
k \\
v
\end{array}\right) e^{i v t}\right\}\right| e^{i t / 2} \mid \\
& \leq \frac{1}{2^{n+k} \pi t}\left|\sum_{k=0}^{n}\left(\begin{array}{l}
n \\
k
\end{array}\right) \operatorname{Re}\left\{\sum_{v=0}^{k}\left(\begin{array}{l}
k \\
v
\end{array}\right) e^{i v t}\right\}\right| \\
& \leq \frac{1}{2^{n+k} \pi t}\left|\sum_{k=0}^{\tau-1}\left(\begin{array}{l}
n \\
k
\end{array}\right) \operatorname{Re}\left\{\sum_{v=0}^{k}\left(\begin{array}{l}
k \\
v
\end{array}\right) e^{i v t}\right\}\right|+\frac{1}{2^{n+k} \pi t}\left|\sum_{k=\tau}^{n}\left(\begin{array}{l}
n \\
k
\end{array}\right) \operatorname{Re}\left\{\sum_{v=0}^{k}\left(\begin{array}{l}
k \\
v
\end{array}\right) e^{i v t}\right\}\right| \\
& =k_{1}+k_{2} \\
& \left|k_{1}\right|=\frac{1}{2^{n+k} \pi t}\left|\sum_{k=0}^{\tau-1}\left(\begin{array}{l}
n \\
k
\end{array}\right) \operatorname{Re}\left\{\sum_{v=0}^{k}\left(\begin{array}{l}
k \\
v
\end{array}\right) e^{i v t}\right\}\right| \\
& \leq \frac{1}{2^{n+k} \pi t}\left|\sum_{k=0}^{\tau-1}\left(\begin{array}{l}
n \\
k
\end{array}\right)\left\{\sum_{v=0}^{k}\left(\begin{array}{l}
k \\
v
\end{array}\right)\right\}\right|\left|e^{i v t}\right| \\
& \leq \frac{1}{2^{n+k} \pi t} \sum_{k=0}^{\tau-1}\left(\begin{array}{l}
n \\
k
\end{array}\right) \sum_{v=0}^{k}\left(\begin{array}{l}
k \\
v
\end{array}\right) \\
& =\frac{1}{2^{n} \pi t} \sum_{k=0}^{\tau-1}\left(\begin{array}{l}
n \\
k
\end{array}\right) \\
& =O\left(\frac{1}{t}\right)
\end{aligned}
$$

Now,

$$
\left|k_{2}\right| \leq \frac{1}{2^{n+k} \pi t}\left|\sum_{k=\tau}^{n}\left(\begin{array}{l}
n \\
k
\end{array}\right) \operatorname{Re}\left\{\sum_{v=0}^{k}\left(\begin{array}{l}
k \\
v
\end{array}\right) e^{i v t}\right\}\right|
$$




$$
\begin{aligned}
& \leq \frac{1}{2^{n+k} \pi t} \sum_{k=\tau}^{n}\left(\begin{array}{l}
n \\
k
\end{array}\right) 0 \leq m \leq k\left|\sum_{v=0}^{\max }\left(\begin{array}{l}
k \\
v
\end{array}\right) e^{i v t}\right| \\
& =O\left(\frac{1}{t}\right)
\end{aligned}
$$

\section{Proof}

Proof of Theorem 1: We have to show, under the hypothesis of the theorem, that

$$
\int_{0}^{\pi} \phi(t) K_{n}(t) d t=o(1), \text { as } n \rightarrow \infty
$$

we set, for $0<\delta<\pi$,

$$
\begin{aligned}
t_{n}^{(E, 1)(E, 1)}-f(x) & =\int_{0}^{\pi} \phi(t) K_{n}(t) d t \\
& =\left[\int_{0}^{1 / n}+\int_{1 / n}^{\delta}+\int_{\delta}^{\pi}\right] \phi(t) K_{n}(t) d t \\
& =I_{1}+I_{2}+I_{3}, \text { say }
\end{aligned}
$$

Now, let

$$
\begin{aligned}
& \left|I_{1}\right|=\int_{0}^{1 / n}|\phi(t)|\left|K_{n}(t)\right| d t \\
& =O(n)\left[\int_{0}^{1 / n}|\phi(t)| d t\right] \text { by Lemma } 1 \\
& =O(n)\left[o\left\{\frac{1}{n \alpha(n)}\right\}\right] \\
& =o\left\{\frac{1}{\alpha(n)}\right\} \\
& =o(1) \text {, as } n \rightarrow \infty \\
& \left|I_{2}\right|=\int_{1 / n}^{\delta}|\phi(t)|\left|K_{n}(t)\right| d t \\
& =O\left[\int_{1 / n}^{\delta} \mid \phi(t)\left(\frac{1}{t}\right) d t\right] \text { by Lemma } 2 \\
& =O\left[\left\{\frac{1}{t} \Phi(t)\right\}_{1 / n}^{\delta}+\int_{1 / n}^{\delta} \frac{1}{t^{2}} \Phi(t) d t\right] \\
& =O\left[o\left\{\frac{1}{\alpha(1 / t)}\right\}_{1 / n}^{\delta}+\int_{1 / n}^{\delta} o\left\{\frac{1}{t \alpha(1 / t)}\right\} d t\right] \\
& =O\left[o\left\{\frac{1}{\alpha(n)}\right\}+\int_{1 / \delta}^{n} o\left\{\frac{1}{u \alpha(u)}\right\} d u\right] \\
& =o\left\{\frac{1}{\alpha(n)}\right\}+o\left\{\frac{1}{n \alpha(n)}\right\} \int_{1 / \delta}^{n} d u
\end{aligned}
$$


Using second-mean value theorem for the integral in the second term as $\alpha(n)$ is monotonic

$$
\begin{aligned}
& =o(1)+o(1) \text { as } n \rightarrow \infty \\
& =o(1) \text { as } n \rightarrow \infty
\end{aligned}
$$

Finally,

$$
\left|I_{3}\right|=\int_{\delta}^{\pi}|\phi(t)|\left|K_{n}(t)\right| d t=o(1) \text { as } n \rightarrow \infty
$$

By using Riemann-Lebesgue theorem and regularity condition of summability.

Combining (5.2), (5.3) and (5.4) we have

$$
t_{n}^{(E, 1)(E, 1)}-f(x)=o(1) \text {, as } n \rightarrow \infty
$$

This completes the proof of theorem 1 .

Proof of Theorem 2:

$$
\begin{aligned}
\tilde{t}_{n}^{(E, 1)(E, 1)}-\tilde{f}(x) & =\int_{0}^{\pi} \psi(t) \tilde{K}_{n}(t) d t \\
& =\left[\int_{0}^{1 / n}+\int_{1 / n}^{\delta}+\int_{\delta}^{\pi}\right] \psi(t) \tilde{K}_{n}(t) d t \\
& =J_{1}+J_{2}+J_{3} \text { (say) }
\end{aligned}
$$

Let

$$
\begin{aligned}
\left|J_{1}\right| & =\int_{0}^{1 / n}|\psi(t)| \tilde{K}_{n}(t) \mid d t \\
& =O\left[\int_{0}^{1 / n} \frac{1}{t}|\psi(t)| d t\right] \text { by Lemma } 3 \\
& =O(n)\left[\int_{0}^{1 / n}|\psi(t)| d t\right] \\
& =O(n)\left[o\left\{\frac{1}{n \alpha(n)}\right\}\right] \\
& =O\left\{\frac{1}{\alpha(n)}\right\} \\
& =o(1), \text { as } n \rightarrow \infty \\
\left|J_{2}\right| & =\int_{1 / n}^{\delta}|\psi(t)| \tilde{K}_{n}(t) \mid d t \\
& =O\left[\int_{1 / n}^{\delta} \frac{1}{t}|\psi(t)| d t\right] \text { by Lemma } 4 \\
& =O\left[\left\{\frac{1}{t} \Psi(t)\right\}_{1 / n}^{\delta}+\int_{1 / n}^{\delta} \frac{1}{t^{2}} \Psi(t) d t\right] \\
& \left.O\left\{\frac{1}{\alpha(1 / t)}\right\}_{1 / n}^{\delta}+\int_{1 / n}^{\delta} o\left\{\frac{1}{t \alpha(1 / t)}\right\} d t\right]
\end{aligned}
$$




$$
\begin{aligned}
& =O\left[o\left\{\frac{1}{\alpha(n)}\right\}+\int_{1 / \delta}^{n} o\left\{\frac{1}{u \alpha(u)}\right\} d u\right] \\
& =o\left\{\frac{1}{\alpha(n)}\right\}+o\left\{\frac{1}{n \alpha(n)}\right\} \int_{1 / \delta}^{n} d u
\end{aligned}
$$

Using second-mean value theorem for the integral in the second term as $\alpha(n)$ is monotonic

$$
\begin{aligned}
& =o(1)+o(1), \text { as } n \rightarrow \infty \\
& =o(1), \text { as } n \rightarrow \infty
\end{aligned}
$$

Finally,

$$
\left|J_{3}\right|=\int_{\delta}^{\pi}|\psi(t)|\left|\tilde{K}_{n}(t)\right| d t=o(1) \text {, as } n \rightarrow \infty
$$

By using Riemann-Lebesgue theorem and regularity condition of summability.

Combining (5.6), (5.7) and (5.8) we have

$$
\tilde{t}_{n}^{(E, 1)(E, 1)}-\tilde{f}(x)=o(1), \text { as } n \rightarrow \infty
$$

This completes the proof of theorem 2 .

\section{Conclusion}

In the field of summability theory, various results pertaining $(E, 1),(E, 1) X$ and $X(E, 1)$ summabilities of Fourier series as well as its Allied series have been reviewed. In future, the present work can be extended to establish new results under certain conditions.

\section{REFERENCES}

[1] Chandra, P. and Dikshit, G.D., On the $|B|$ and $|E, q|$ summability of a fourier series,its conjugate series and their derived series, Indian J. pure appl. Math., 12(11) 1350-1360, (1981).

[2] Chandra, P. On the $|E, q|$ summability of a Fourier series and its conjugate series. Riv. Mat. Univ. Parma (4), 3, 65-78 (1977).

[3] Dhakal, Binod Prasad, Approximation of function belonging to lip $(\alpha, p)$ class by $(E, 1)\left(N, p_{n}\right)$ mean of its fourier series, Kathmandu University Journal of Science, Engg. And Technology 7, 1 8 (2011).

[4] Hardy, G.H. "Divergent Series”, Oxford (1949).

[5] Nigam, H.K. and Sharma, Ajay, On $(N, p, q)(E, 1)$ summablity of fourier series, IJMMS, Vol.2009, (2009).

[6] Prasad Kanhaiya, On the $\left(N, p_{n}\right) C_{1}$ summability of a sequence of fourier coefficients, Indian J. pure appl. Math., 12(7) 874-881, (1981).

[7] Titchmarsh, E.C. "The Theory of functions", Oxford (1952).

[8] Tiwari, Sandeep Kumar and Bariwal Chandrashekhar, Degree of Approximation of function belonging to the Lipschitz class by $(E, q)(C, 1)$ mean of its fourier series, IJMA 1 (1), 2-4, (2010).

[9] Zygmund, A. “Trigonometrical Series”, Vol. I and II, Warsaw (1935). 